\title{
Inhalationstrauma: Prognose meist gut, außer bei Brandunfällen
}

\author{
Verbrennungsopfer erleiden häufig ein Inhalationstrauma, die Prognose ist dann schlechter. Die \\ hohe Frühmortalität dieser Patienten liegt vorrangig an der Intoxikation mit Erstickungsgasen.
}

Patienten mit Inhalationstrauma durch toxische Gase haben in der Regel eine gute Prognose; eine Ausnahme stellen Inhalationstraumata bei einem Brandunfall dar. 80 Prozent der Todesfälle seien durch das Inhalationstrauma verursacht, berichtete Dr. Sandra Riedel vom Universitätsklinikum Magdeburg.

20 bis 30 Prozent der Opfer von Brandunfällen erleiden auch ein Inhalationstrauma, so Riedel. Die Mortalität dieser Patienten liegt bei $10 \%$, haben sie zusätzlich schwere Verbrennungen steigt sie auf mehr als $50 \%$. Die Schädigung kann auf toxischem Weg durch Reizgase, thermisch wie auch durch systemische Intoxikation durch asphyktische Gase erfolgen. Ausmaß und Ort der Schädigung durch Reizgase sind abhängig von der Wasserlöslichkeit der inhalierten Substanzen - gut wasserlösliche seien oft hoch irritativ, lipidlösliche erreichen die unteren Atemwege - der Partikelgröße (>10 $\mu$ m verbleiben in Nase und Rachen, $<5 \mu$ m erreichen die Alveolen), Dauer und Ort der Exposition sowie den Komorbiditäten der Betroffenen.

\section{Ursachen von Inhalationstraumata}

Allgemein sei die Prognose bei einem Inhalationstrauma ohne Brandunfall sehr gut, berichtete Riedel. Typische Symptome, zum Beispiel bei Chlorexpositon bei Swimmingpool-Arbeit, sind Augen- und Schleimhautreizungen, Stridor, Husten, Bronchospasmen und Dyspnoe. Über 90 Prozent der Betroffenen würden komplett gesund, sagte Riedel, fünf bis sechs Prozent drohten allerdings Langzeitfolgen wie RADS (Reactive Airway Disease Syndrome), Bronchiolitis und Bronchiektasen.

Unterschieden werden bei den Reizgasen toxisch-irritative wie Ammoniak, Chlor-, Fluor- und Schwefelwasserstoff, die vor allem die oberen Atemwege schädigen und bei massiver Exposition auch ein Lungenödem auslösen können. Lipophile Substanzen wie Aldehyde, Stickstoffoxide und Phosgen erreichen gut die unteren Atemwege und führen nach Angaben von Riedel zum Beispiel zu schwerer ödematöser Bronchitis/Bronchiolitis.

\section{Besondere Gefahr - Erstickungsgase}

Besonders riskant ist bei Brandunfällen die Freisetzung von Erstickungsgasen wie Kohlendioxid, Kohlenmonoxid und Zyanidin, das etwa bei Verbrennung von Polyurethan, Nylon und Wolle entsteht. Die Symptome einer CO-Intoxikation sind unspezifisch und reichen von Dyspnoe, neurologischen Beschwerden (Kopfschmerzen, Verwirrtheit bis zum Koma) bis zu kardialen Symptomen (Herzinsuffizienz, Arrhythmien). Die typische „kirschrote“ Haut entstehe erst bei einem CO-Hb $>40 \%$, sagte Riedel. Bei Inhalation von Zyanid kommt es rasch zur Bewusstlosigkeit und bei ausbleibender adäquater Therapie zum schnellen Tod der Patienten.

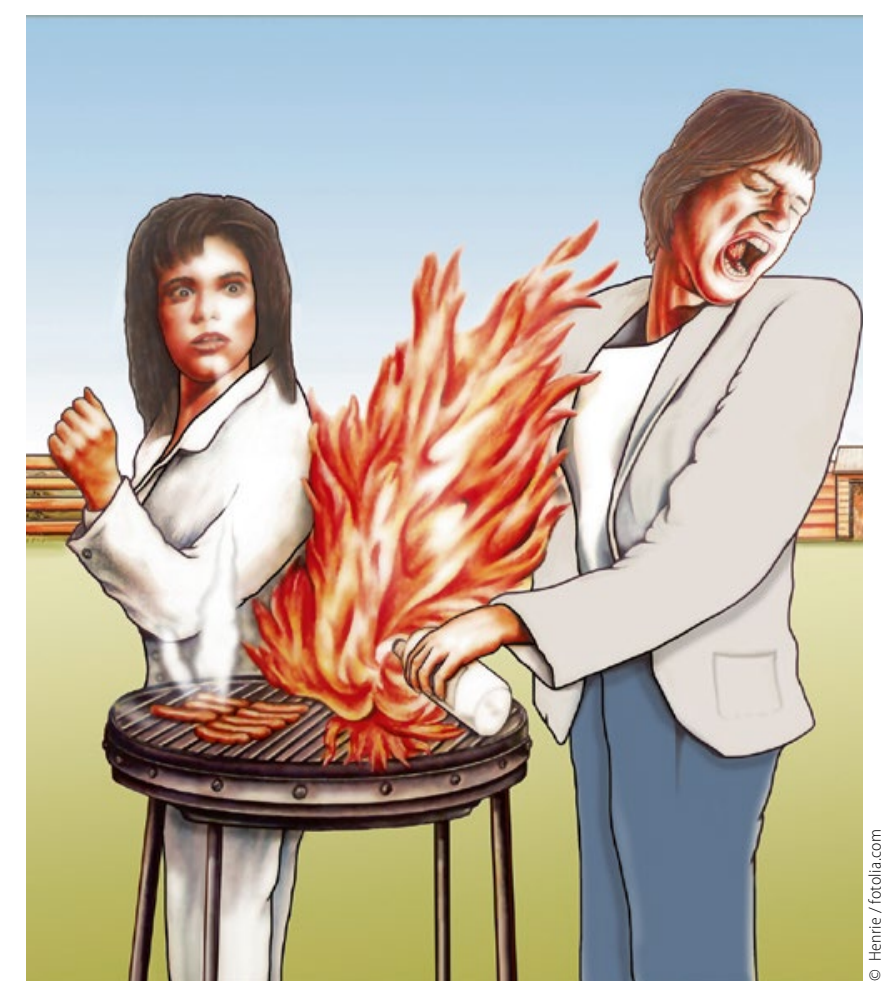

Brandunfälle beim Grillen sind keine Seltenheit. Inhalationstraumata gehen dabei mit einer hohen Akutsterblichkeit einher.

\section{Welche Sofortmaßnahmen?}

$\mathrm{Zu}$ den Sofortmaßnahmen zählen bei allen Inhalationstraumata eine Sauerstoffgabe $\left(100 \% \mathrm{FiO}_{2}\right)$ und bei Bronchospasmus Betamimetika inhalativ, sagte Riedel. Für die häufig praktizierte Kortikoidgabe (inhalativ oder systemisch) gebe es hingegen keine Evidenzen für einen Benefit, sie werde daher nicht mehr empfohlen. Vernebeltes Heparin - in Kombination mit Acetylcystein - sei Studien zufolge vielversprechend, aber noch kein Standard. Auch gebe es keinen verlässlichen Indikator, der für oder gegen eine Intubation spreche.

Im Falle einer CO-Intoxikation besteht außerdem die Option einer hyperbaren Oxygenierung in der Druckkammer, während bei einer Zyanidintoxikation die gezielte Antidot-Gabe mit Hydroxycobalamin möglich ist.

Roland Fath

Kongress der Deutschen Gesellschaft für Pneumologie und Beatmungsmedizin (DGP), 3.-5. März 2016, Leipzig 